\title{
WWC3 inhibits intimal proliferation following vascular injury via the Hippo signaling pathway
}

\author{
BEIJIA CHEN and GUINAN LIU \\ Department of Cardiology, The First Affiliated Hospital of The China Medical University, \\ Shenyang, Liaoning 110001, P.R. China
}

Received December 17, 2016; Accepted November 28, 2017

DOI: $10.3892 / \mathrm{mmr} .2018 .8484$

\begin{abstract}
The Hippo signaling pathway is involved in the formation and development of the cardiovascular system. In the present study, the effects of WWC family member 3 (WWC3) on vascular smooth muscle cells (VSMCs) following injury were investigated, in addition to the associated mechanisms underlying this process. Platelet-derived growth factor BB (PDGF-BB) was used as a cell injury factor, and rats with balloon injuries were used as a model of carotid intimal injury. Furthermore, the expression levels of WWC3 in VSMCs and arteries post-injury were investigated, in addition to the effect of WWC3 on the proliferation and migration of VSMCs. The results demonstrated that following injury, WWC3 expression was suppressed in VSMCs and the rat carotid artery, and the activity of the Hippo signaling pathway was significantly downregulated. In addition, the expression of YY1-associated protein-1 (YAP) and a number of its downstream target genes, including connective tissue growth factor (CTGF), were enhanced, thus enhancing the proliferation and migration of VSMCs. Knockdown of WWC3 suppressed the levels of large tumor suppressor kinase 1 (LATS1) expression and YAP phosphorylation, and the expression of YAP, CTGF and cyclin E was subsequently enhanced, thus promoting cell proliferation and migration. Similar results were obtained following overexpression of WWC3. Treatment with PDGF-BB was revealed to suppress the proliferation and migration of VSMCs transfected with the WWC3 plasmid, compared with VSMCs transfected with an empty vector. The present study demonstrated that
\end{abstract}

Correspondence to: Professor Guinan Liu, Department of Cardiology, The First Affiliated Hospital of The China Medical University, 155 Nanjing North Street, Shenyang, Liaoning 110001, P.R. China

E-mail: guinanliu@hotmail.com

Abbreviations: VSMC, vascular smooth muscle cells; PDGF-BB, platelet-derived growth factor BB; YAP, YY1-associated protein; CTGF, connective tissue growth factor; LATS, large tumor suppressor

Key words: WW domain-containing protein-3, Hippo signaling pathway, smooth muscle cells, vascular injury
WWC3 may interact with LATS1 in order to upregulate the Hippo signaling pathway via co-immunoprecipitation and enhancement of the phosphorylation of LATS1, in addition to the corresponding suppression of the nuclear import of YAP. However, VSMCs transfected with WWC3 plasmid with a deletion of the WW domain fail to exhibit this effect. These results suggested that WWC3 expression is downregulated in VSMCs during neointimal hyperplasia following injury (PDGF-BB stimulation or balloon injury). WWC3 upregulates the activity of the Hippo signaling pathway, and weakens the proliferation and migration of VSMCs. Furthermore, the results of the present study suggested that WWC3 may interact with LATS1 to promote the phosphorylation of YAP and reduce its nuclear translocation, upregulate the activity of the Hippo pathway, and suppress the proliferation and migration of VSMCs following injury.

\section{Introduction}

Atherosclerosis, hypertension, diabetes and various other vascular remodeling diseases pose serious threats to human health. Restenosis (RS), following percutaneous coronary intervention, is a pathogenic vascular remodeling disease. At present, intra-intima migration and proliferation of vascular smooth muscle cells (VSMCs) are considered to be among the most prominent pathogeneses associated with vascular remodeling (1). Considering the high rates of morbidity and pathogenesis associated with $\mathrm{RS}$, the studies regarding the negative effects of long-term therapeutics on RS have been extensively performed. The underlying mechanisms of VSMC proliferation, migration and extracellular matrix production post-injury are highly complex and have not yet been completely determined (2). Therefore, advancements with regards to the understanding of the mechanisms associated with post-injury vascular remodeling are of importance, particularly with regards to the development of preventative and therapeutic treatments for cardiovascular diseases.

The Hippo signaling pathway was originally discovered in Drosophila during functional genetic screening (3). The Hippo signaling pathway is a kinase chain, which is composed of a series of protein kinases and transcriptional co-activators, and is involved in organ size regulation, cell proliferation and apoptosis (4-6). The Hippo signaling pathway is highly conserved; however, a similar signaling 
pathway exists in mammals. The Hippo, Salvador, Warts, Mats, Yorki and Scalloped genes in Drosophila are respectively homologous to macrophage stimulating 1 (MST1), salvador family WW domain containing protein 1 (WW45), large tumor suppressor kinases 1 and 2 (LATS1/2), MOB kinase activator 1 (Mob1), YY1 associated protein (YAP)/tafazzin (TAZ) and TEA domain transcription factors (TEAD) in mammals (7). The aforementioned intermolecular interactions are responsible for the workings of mammalian cell signaling pathways, in addition to the mediation of cell proliferation and apoptosis levels in order to regulate organismal growth and development (7). The heart of the Hippo pathway comprises a core kinase cassette that consists of a pair of related serine/threonine kinases, mammalian STE20-like protein kinase 1 (MST1; also known as STK4) and MST2 (also known as STK3), which are homologues of D. melanogaster Hippo 6-9 and LATS1 and LATS2 (1-3), together with the adaptor proteins Salvador homologue 1 (SAV1)1, 10, Mob1A and Mob1B. These proteins limit tissue growth by facilitating LATS1- and LATS2-dependent phosphorylation of the homologous oncoproteins YAP (encoded by $Y A P 1$ ) and transcriptional co-activator with PDZ-binding motif TAZ (also known as WWTR1) (8). YAP is transported to the nucleus following inactivation of the Hippo signaling pathway, which is associated with the activity of numerous intracellular kinases and the activation of the proteasome system. Upstream phosphorylation cascades may phosphorylate YAP, and phosphorylated YAP (p-YAP) is subsequently retained in the cytoplasm via interaction with 14-3-3 protein. Following this, p-YAP is degraded by ubiquitin-dependent proteasomes, thus inhibiting gene expression associated with the promotion of cell proliferation and resistance to apoptosis (9). In conclusion, the Hippo signaling pathway regulates YAP/TAZ function depending on the regulation of its upstream molecules and kinases.

Previous studies have revealed that neointimal hyperplasia-associated diseases, including atherosclerosis, share common underlying molecular mechanisms with tumorigenesis (10). Previous studies have additionally revealed that the Hippo signaling pathway is associated with embryogenesis and the development of the cardiovascular system (11). YAP, the effector of Hippo pathway, is additionally implicated in the phenotypic modulation of smooth muscle $(12,13)$.

WWC family member 3 (WWC3) is a member of the WWC protein family. Limited studies focusing entirely upon investigation of WW and $\mathrm{C} 2$ domain containing 1 (WWC1) have revealed that WWC1 may regulate the Hippo signaling pathway via interaction with the LATS1 WW domain in order to induce the phosphorylation of LATS1 $(14,15)$. Members of the WWC protein family share a number of highly conserved molecular structures, including the WW, C2 and ADDV domains (16). Furthermore, in the present study it has been suggested that WWC3 forms a complex with LATS1, via interaction with the WW domain, in order to regulate the Hippo signaling pathway. Therefore, it is possible that WWC3 may form a complex with LATS1, via its WW domain, in order to regulate the Hippo signaling pathway.

The present study aimed to investigate the expression pattern of WWC3 in VSMCs following vascular injury, the effects of WWC3 expression on the Hippo signaling pathway, and the potential underlying molecular mechanisms associated with this process.

\section{Materials and methods}

Cell culture. Rat A10 aortic VSMCs were purchased from the American Type Culture Collection (Manassas, VA, USA) and cultured in Dulbecco's modified Eagle's medium (Thermo Fisher Scientific, Inc., Waltham, MA, USA) containing $10 \%$ fetal bovine serum (FBS; Invitrogen; Thermo Fisher Scientific, Inc., USA), in a humidified atmosphere of $5 \% \mathrm{CO}_{2}$ at $37^{\circ} \mathrm{C}$. In order to perform platelet-derived growth factor BB (PDGF-BB) experiments, rat VSMCs were grown to $80-90 \%$ confluence, serum-starved for $24 \mathrm{~h}$ and treated with recombinant human PDGF-BB (10 ng/ml; PeproTech, Inc., Rocky Hill, NJ, USA) for a further $48 \mathrm{~h}$. Cells treated with PBS served as the control.

Animal model. Twenty male Wistar rats (350-400 g, 10 weeks) were purchased from the Experimental Animal Center (China Medical University, Shenyang, China). Housing conditions were: Temperature, $24^{\circ} \mathrm{C}$, light/dark cycle (12-h), food (LAD 2001; Trophic Animal Feed High-Tech Co., Ltd., Nantong, China) and sterilized water provided. All animal studies were granted ethical approval by the Institutional Animal Care and Use Committee. Following this, rat carotid artery balloon injury was performed on 10 rats as previously described and the 10 remaining rats were the controls (17). The left common, external and internal carotid arteries were dissected, and vascular injury was administered to the left common carotid artery using an A Baxter 2-Fr balloon catheter (Baxter International, Deerfield, IL, USA). Following this, the balloon catheter was removed, the external carotid artery was ligated and the wound was subsequently closed. The rats were administered penicillin to prevent infection and were then sacrificed at 14 days post-injury. The right carotid artery served as an uninjured control.

Plasmid construction and transfection. Empty p-enhanced green fluorescent protein (EGFP)-C2, pEGFP-C2-WWC3 and pEGFP-C2- $\Delta \mathrm{WW}$ vectors were obtained from the University of Münster (Münster, Germany). pGL3b_8xGTIIC-luciferase and pRL-TK $1 \mu \mathrm{g}$ and $50 \mathrm{ng}$, respectively plasmids were additionally purchased (Addgene, Inc., Cambridge, MA, USA). Lipofectamine ${ }^{\circledR} 3000$ (Invitrogen; Thermo Fisher Scientific, Inc.) transfection reagent was used for plasmid transfection. The density of cells transfected with 2 pmol siRNA-WWC3, (Santa Cruz Biotechnology, Inc., Dallas, TX, USA) was $80-90 \%$. A total of $48 \mathrm{~h}$ later, cells were harvested for the further experiments.

Hematoxylin and eosin $(H \& E)$ staining and immunohistochemistry. The common carotid arteries were fixed with $10 \%$ neutral formalin for $12 \mathrm{~h}$ at $25^{\circ} \mathrm{C}$, embedded in paraffin and cut into sections $(4-\mu \mathrm{m})$ and, following this, the sections were deparaffinized using xylene and rehydrated through a graded alcohol series (100-95-85-75\%). H\&E staining was performed following a standard protocol (18). Immunostaining was performed using the avidin-biotin-peroxidase complex method (cat. no. KIT-9710; Ultrasensitive ${ }^{\mathrm{TM}}$; Fuzhou Maixin 
Biotech Co., Ltd., Fuzhou, China). Normal goat serum (cat. no. KIT-9710, Ultrasensitive ${ }^{\mathrm{TM}}$; Fuzhou Maixin Biotech Co., Ltd.) was used to reduce nonspecific binding at $25^{\circ} \mathrm{C}$ for $30 \mathrm{~min}$. The primary antibodies used were as follows: WWC3 (cat. no. HPA039814; 1:200; Merck KGaA, Darmstadt, Germany), YAP (cat. no. 14074; 1:100; Cell Signaling Technology, Inc., Danvers, MA, USA), connective tissue growth factor (CTGF) and cyclin E (cat. nos. sc-25440 and sc-481; 1:100; Santa Cruz Biotechnology, Inc.) at $4^{\circ} \mathrm{C}$ overnight. Staining for primary antibody was performed at room temperature for 2 h. Biotinylated serum IgG (cat. no. KIT-9710, ready-to-use; Fuzhou Maixin Biotech Co., Ltd.,) was used as the secondary antibody. Following rinsing in PBS, the sections were incubated with horseradish peroxidase-conjugated streptavidin-biotin, followed by 3,3'-diaminobenzidine tetrahydrochloride to develop the peroxidase reaction. The sections were counterstained with hematoxylin at $25^{\circ} \mathrm{C}$ for 5 min and dehydrated in ethanol before mounting. All slides were examined per view at magnification, $\mathrm{x} 400$.

Protein extraction and western blotting analysis. VSMCs were washed using PBS and collected for protein extraction. The rat carotid artery was dissected into small pieces and cells were lysed using a homogenizer in cold radioimmunoprecipitation assay lysis buffer (Santa Cruz Biotechnology, Inc.). Following sonication (frequency: $30 \%$ of maximum, at $4^{\circ} \mathrm{C}$ for $5 \mathrm{sec})$ and centrifugation $\left(12,000 \mathrm{x} \mathrm{g}, 4^{\circ} \mathrm{C}, 15 \mathrm{~min}\right)$ of the cell lysate, protein samples were quantified using ultraviolet spectrophotometry and loaded (40 $\mu \mathrm{g} /$ lane) onto a $10 \%$ SDS-PAGE gel. The following primary antibodies were used in said analyses: WWC3 (cat. no. HPA039814; 1:500; Merck KGaA), YAP (cat. no. 14074; 1:500; Cell Signaling Technology, Inc.), p-YAP (cat. no. 13008; 1:500; Cell Signaling Technology, Inc.), phospho-LATS1 (cat. no. 8654; 1:500; Cell Signaling Technology, Inc.), CTGF and cyclin E (cat. nos. sc-25440 and sc-481; 1:200; Santa Cruz Biotechnology, Inc.), GFP-tag (cat. no. 66002-1-Ig; 1:2,000; Wuhan Sanying Biotechnology, Wuhan, China), $\beta$-actin (cat. no. sc-47778; 1:1,000; Santa Cruz Biotechnology, Inc., USA), $\alpha$-tubulin (cat. no. sc-5286; 1:1,000; Santa Cruz Biotechnology, Inc.) and lamin B1 (cat. no. ab16048; 1:1,000; Abcam, Cambridge, MA, USA) incubated at $4^{\circ} \mathrm{C}$ overnight. Samples were incubated with peroxidase-coupled secondary antibodies (cat. nos. sc-516102 and sc-2007; 1:2,000, Santa Cruz Biotechnology, Inc.) at $25^{\circ} \mathrm{C}$ for $2 \mathrm{~h} .5 \%$ skimmed milk (BD Biosciences, Franklin Lakes, NJ, USA) was used for blocking at $25^{\circ} \mathrm{C}$ for $1 \mathrm{~h}$. Proteins were transferred to polyvinylidene difluoride membranes and visualized using an enhanced chemiluminescence kit (GE Healthcare, Chicago, IL, USA). Images were obtained using a BioRad Imaging System (Bio-Rad Laboratories, Inc., Hercules, CA, USA). The relative protein levels were quantified with respect to the use of $\beta$-actin, $\alpha$-tubulin and lamin B1 proteins as loading controls.

Immunoprecipitation. Supernatants of the cell lysate were collected following centrifugation, $\left(12,000 \mathrm{x} \mathrm{g}, 15 \mathrm{~min}, 4^{\circ} \mathrm{C}\right)$ precleared with protein $\mathrm{G}$-agarose for $2 \mathrm{~h}$ at $4^{\circ} \mathrm{C}$ and incubated with the aforementioned antibodies overnight at $4^{\circ} \mathrm{C}$. LATS1 (5 $\mu \mathrm{g} / \mathrm{ml}$ for IP; Santa Cruz Biotechnology, Inc.), WWC3 (1:500 for immunoblotting; Sigma-Aldrich; Merck KGaA, Darmstadt, Germany). Wild-type immunoglobulin G was used as a negative control. Then detected by western blotting analysis. Then LATS1 and WWC3 were measured by western blotting analysis according to the aforementioned protocol. Cell lysates from A10 cells were subjected to immunoprecipition with anti-GFP antibody GFP-tag (cat. no. JL-8; 1:3,000; Clontech Laboratories, Inc., Mountainview, CA, USA) or control IgG (cat. no. A7028; $2 \mu \mathrm{g} / \mathrm{ml}$; Beyotime Institute of Biotechnology, Shanghai, China), and the presence of LATS1 was examined by anti-WWC3 immunoblotting.

Immunofluorescence staining. Cells were fixed with $4 \%$ paraformaldehyde for $15 \mathrm{~min}, 25^{\circ} \mathrm{C}$, and blocked using $5 \%$ goat serum (Invitrogen, Thermo Fisher Scientific, Inc.) for $2 \mathrm{~h}$ at room temperature. Cells were incubated with the following primary antibodies: WWC3 (cat. no. HPA039814; 1:50; Sigma-Aldrich; Merck KGaA), LATS1 (cat. no. sc-398560; anti-mouse; 1:50; Santa Cruz Biotechnology, Inc.), YAP (cat. no. 14074; 1:50; Cell Signaling Technology, Inc.) overnight at $4^{\circ} \mathrm{C}$. Subsequently, the cells were incubated with secondary antibodies for $1 \mathrm{~h}$ at room temperature (anti-rabbit secondary antibody and anti-mouse secondary antibody; cat. nos. A11008 and A32727; 1:200; Invitrogen; Thermo Fisher Scientific, Inc.). Cell nuclei were counterstained using DAPI (cat. no. D9542; Sigma-Aldrich; Merck KGaA), and images were captured using a confocal microscope at magnification, x400 (Olympus, Corporation, Tokyo, Japan).

MTT assay. Cells were plated in 96-well plates in medium containing $10 \% \mathrm{FBS}$ at $\sim 3,000$ cells/well, and the cells were then harvested for further experimentation at 24, 48, 72, 96, 120 -h time intervals. Cell viability was determined using an MTT assay. A total of $20 \mu 15 \mathrm{mg} / \mathrm{ml}$ MTT solution (cat. no. M2128; Sigma-Aldrich; Merck KGaA) was added to each well, and the plates were incubated for $4 \mathrm{~h}$ at $37^{\circ} \mathrm{C}$. The medium from each well was removed and $150 \mu \mathrm{l}$ dimethyl sulfoxide (cat. no. D2650; Sigma-Aldrich; Merck KGaA) was added to dissolve the purple formazan. The results were quantified using spectrophotometry at a wavelength of $550 \mathrm{~nm}$.

Cell invasion assay. A 24-well Transwell chamber with a pore size of $8-\mu \mathrm{m}$ was used in order to determine cell invasion (Costar; Corning Incorporated, Corning, NY, USA). A total of $5 \times 10^{4}$ cells were trypsinized and transferred to the upper Matrigel chamber containing $100 \mu \mathrm{l}$ serum-free medium, and incubated for $16 \mathrm{~h}$ at $25^{\circ} \mathrm{C}$. Medium containing $10 \% \mathrm{FBS}$ was added to the lower chamber to act as the chemoattractant. Following this, the non-invaded cells present on the upper membrane surface were removed using a cotton swab, and the cells on the bottom surface were fixed using $4 \%$ paraformaldehyde at $25^{\circ} \mathrm{C}$ for $20 \mathrm{~min}$ and stained using hematoxylin at $25^{\circ} \mathrm{C}$ for $10 \mathrm{~min}$. The numbers of invaded cells were counted in five randomly selected, high power fields using a light microscope at a magnification, x200 (Olympus Corporation, Tokyo, Japan).

Scratch wound healing assay. VSMCs were seeded into 6-well plates at a confluent density $\left(110^{6}\right.$ cells/well). Mitomycin C (20 $\mu \mathrm{mol} / \mathrm{l}$; Sigma-Aldrich; Merck KGaA) was added into each well, and the plates were incubated for $2 \mathrm{~h}$ at $25^{\circ} \mathrm{C}$. Following this, cell migration was assayed $24 \mathrm{~h}$ post-scratching with a $100 \mu \mathrm{l}$ pipette micro tip, and the relative closure distances were 
A

Control

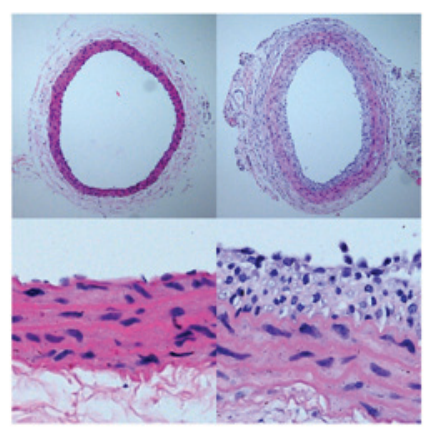

C
B

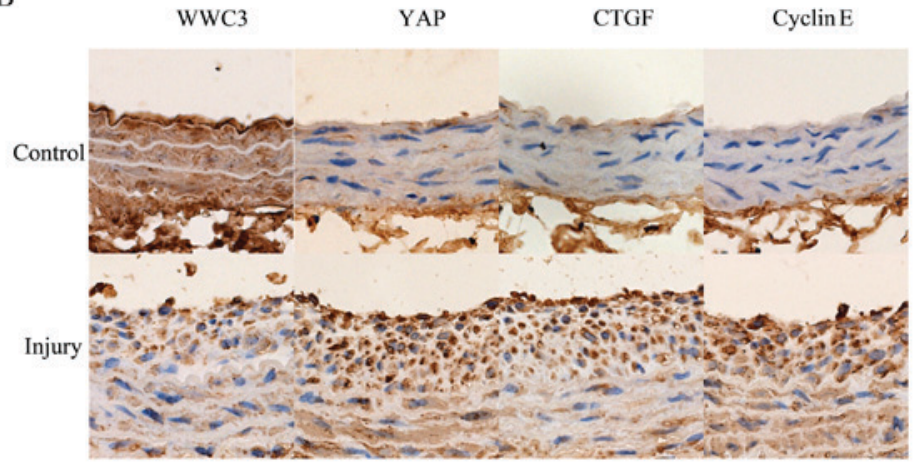

Injury

D

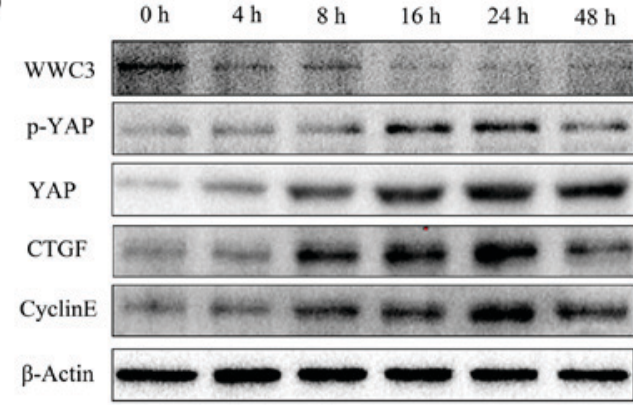

H
$\mathrm{E}$

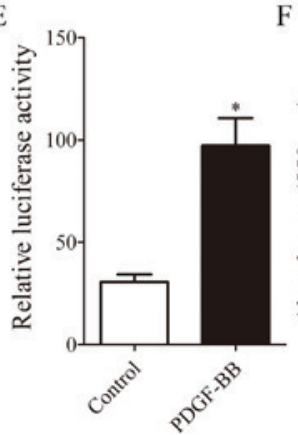

$\mathrm{F}$

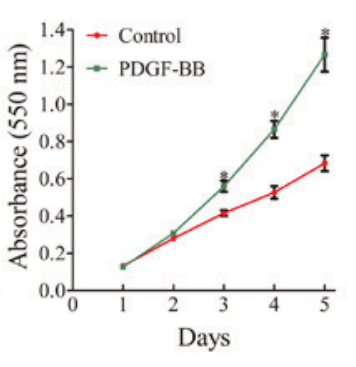

G

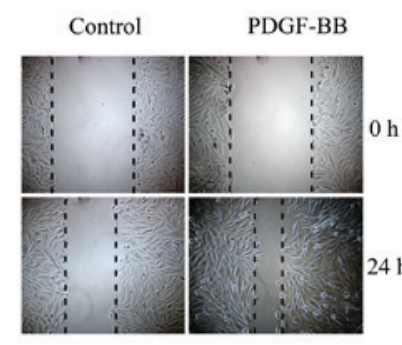

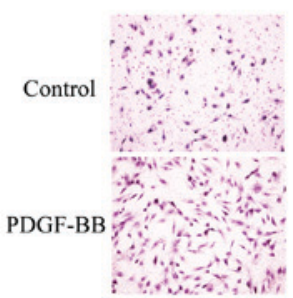

Figure 1. Suppression of WWC3 expression in VSMCs is induced by treatment with PDGF-BB and balloon injury. (A) Hematoxylin and eosin staining of carotid artery tissues obtained from either control or balloon-injured vessels. I and II (magnification, x100), III and IV (magnification, x400). (B) The expression levels of WWC3, YAP, CTGF and cyclin E in rat carotid arteries following balloon injury, revealed by immunohistochemistry (magnification, x400). (C) At 14 days post-injury, rat controls or balloon-injured carotid arteries were harvested for western blot analysis. (D) VSMCs treated with PDGF-BB (10 ng/ml) for different time periods were harvested for western blot analysis. (E) The transcriptional activity of the TEA domain transcription factor was measured using a dual-luciferase reporter assay following treatment with PDGF-BB for $24 \mathrm{~h}$. (F) The MTT assay demonstrated that treatment with PDGF-BB promotes VSMC proliferation. (G) Scratch wound healing assays and $(\mathrm{H})$ Transwell chamber assays revealed that treatment with PDGF-BB promotes VSMC migration. The results are presented as the mean \pm standard deviation of three independent experiments. ${ }^{*} \mathrm{P}<0.05$ vs. control. VSMCs, vascular smooth muscle cells; WWC3, WW family member 3 ; PDGF-BB, platelet-derived growth factor BB; YAP, YY1-associated protein; p-YAP, phosphorylated YAP; CTGF, connective tissue growth factor.

determined. Five random fields were measured by capturing images using a light microscope at magnification, x200 (Olympus Corporation).

Dual-luciferase reporter assay. VSMCs were seeded in 24-well plates, and transiently transfected with either PGL3b-8x plasmid GTIIC (cat. no. 34615), indicating TEAD transcriptional activity, pRL-TK, WWC3 plasmids or empty plasmids, using Lipofectamine ${ }^{\circledR} 3000$ (Invitrogen; Thermo Fisher Scientific, Inc.). Following a 48-h transfection time period, cells were lysed and luciferase activity was determined using the Dual-Luciferase Assay kit (Promega Corporation, Madison, WI, USA). The activity of thymidine kinase Renilla served as an internal standard for normalization.

Statistical analysis. The statistical software SPSS 17.0 (SPSS, Inc., Chicago, IL, USA) was used for all analyses. All data are expressed as the mean \pm standard deviation. All experiments were replicated triplicate and were analyzed using either the Student's t-test, or one-way analysis of variance followed by a Dunnett-t test as a post-hoc test. $\mathrm{P}<0.05$ was considered to indicate a statistically significant difference.

\section{Results}

Suppression of WWC3 expression in VSMCs is induced by $P D G F-B B$ treatment and balloon injury. Marked neointimal hyperplasia and lumen narrowing were observed using $\mathrm{H} \& \mathrm{E}$ staining 14 days post-injury in the balloon-injured rat carotid artery, compared with the control group (Fig. 1A). Suppression of WWC3 expression and enhancement of YAP, CTGF and cyclin E expression was determined using immunohistochemistry and western blotting analysis (Fig. 1B and C). In addition, it was also revealed that WWC3 expression was 

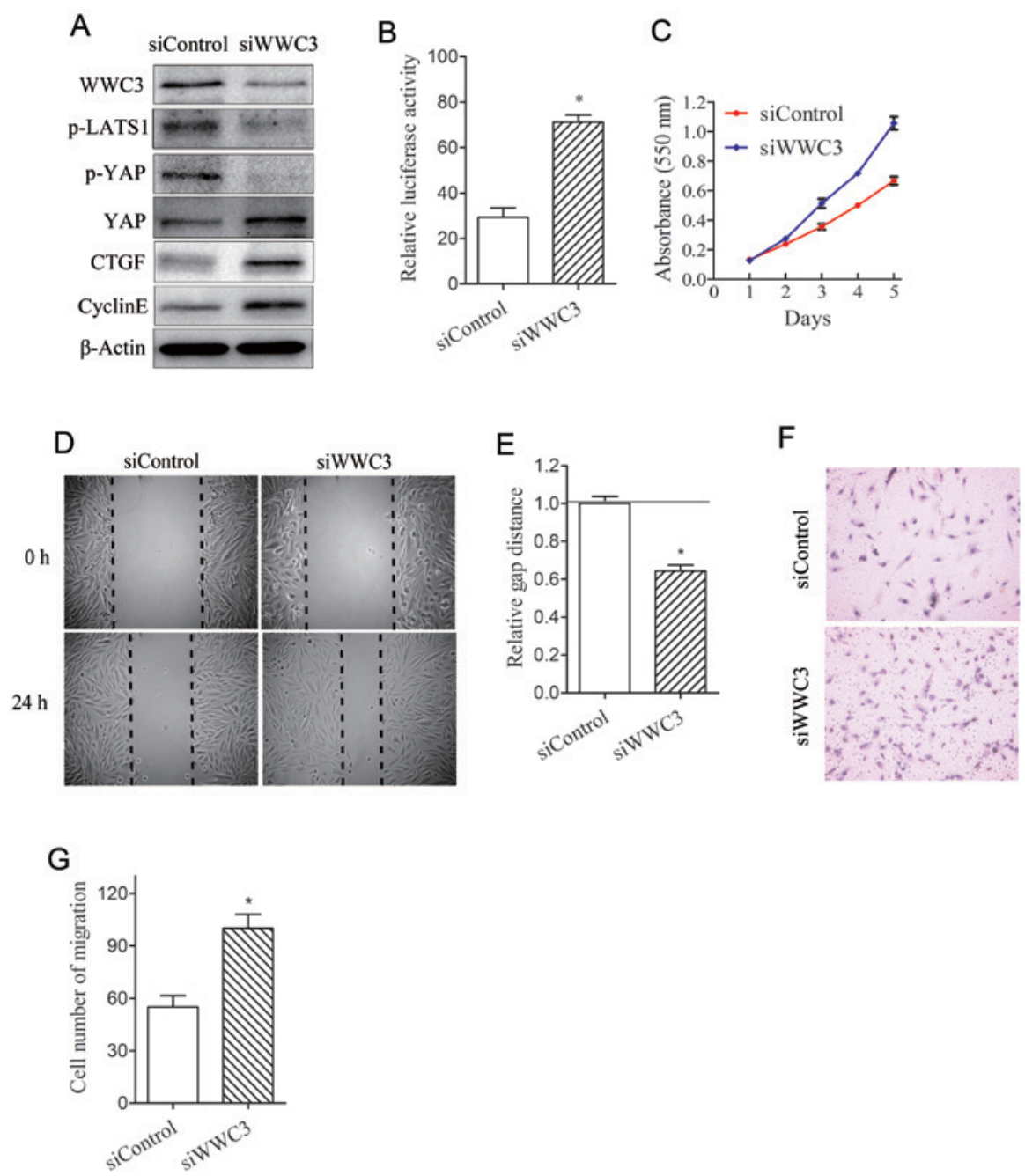

Figure 2. Knockdown of WWC3 promotes VSMC proliferation and migration via downregulation of the Hippo signaling pathway. (A) Western blot analysis for the determination of the protein expression levels of WWC3, p-LATS1, YAP, p-YAP, CTGF and cyclin E in WWC3-knockdown VSMCs. (B) Gene knockdown of WWC3 was performed using siWWC3, and the dual-luciferase activity assay was performed in order to determine the transcriptional activity of TEA domain transcription factors. (C) An MTT assay was used in order to determine cell proliferation; (D) a scratch wound healing assay was performed and (E) analyzed, and a (F) Transwell chamber assay was performed and (G) analyzed in order to determine cell migration. The results are presented as the mean \pm standard deviation of three independent experiments. "P<0.05 vs. control. VSMCs, vascular smooth muscle cells; WWC3, WW domain-containing protein-3; PDGF-BB, platelet-derived growth factor BB; YAP, YY1-associated protein; p-YAP, phosphorylated YAP; p-LATS1, phosphorylated large tumor suppressor kinase 1; CTGF, connective tissue growth factor; si, small interfering RNA.

suppressed in VSMCs treated with PDGF-BB $(10 \mathrm{ng} / \mathrm{ml})$ for $24 \mathrm{~h}$, whereas YAP, p-YAP, CTGF and cyclin E levels were markedly enhanced (Fig. 1D). The in vitro results were consistent with the results of the animal experiments. The transcriptional activity of TEAD suggested that the activity of the Hippo signaling pathway was significantly enhanced following treatment with PDGF-BB (Fig. 1E). Furthermore, VSMC proliferation was determined, via the MTT assay, to be enhanced following treatment with PDGF-BB (Fig. 1F). In addition, the scratch wound healing assay and the Transwell chamber assay revealed that VSMC migration was markedly enhanced following PDGF-BB-induced injury (Fig. $1 \mathrm{G}$ and $\mathrm{H}$ ).

WWC3 expression inhibits the proliferation and migration of VSMCs via upregulation of the Hippo signaling pathway. In order to investigate the association between WWC3 and the Hippo signaling pathway following treatment with
PDGF-BB, the expression of WWC3 was regulated in a bidirectional manner in order to investigate the effects upon the predominant components of the Hippo signaling pathway. Knockdown of WWC3 in VSMCs was revealed to suppress the levels of p-LATS1 and p-YAP, and to enhance the expression of YAP, CTGF and cyclin E (Fig. 2A). The transcriptional activity of TEAD, an indicator of the Hippo-YAP signaling pathway, was demonstrated to be significantly enhanced following suppression of WWC3 (Fig. 2B). Furthermore, VSMC proliferation and migration were enhanced following suppression of WWC3 (Fig. 2C-G); whereas VSMC proliferation and migration were suppressed following overexpression of WWC3 (Fig. 3). In addition, VSMCs were treated with PDGF-BB following overexpression of WWC3, and it was demonstrated that PDGF-BB did not induce a marked effect on the expression of genes associated with the Hippo signaling pathway, VSMC migration and proliferation (Fig. 3A-G). Additionally, in cells transfected with WWC3, 
A

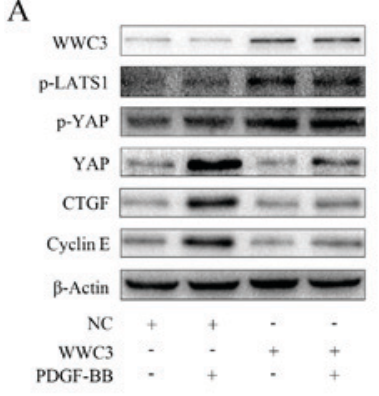

D

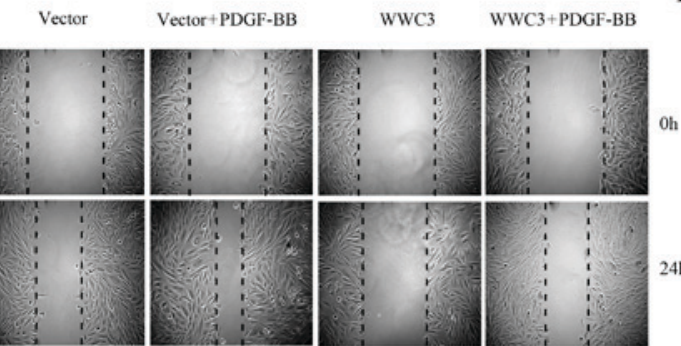

$\mathrm{F}$

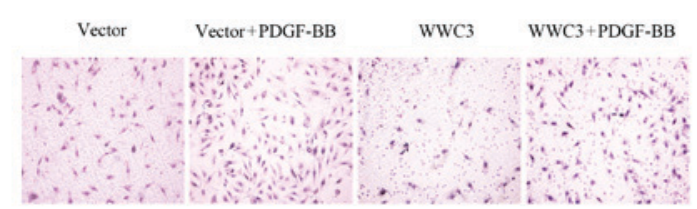

B
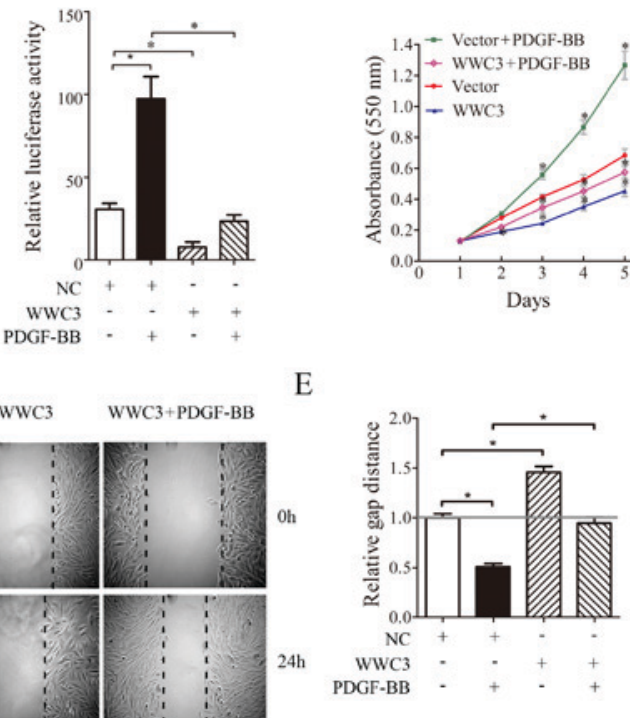

G

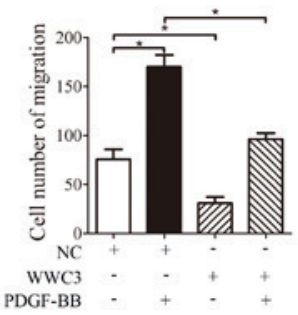

Figure 3. WWC3 inhibits VSMC proliferation and migration by upregulating the activity of the Hippo signaling pathway. (A) Western blotting was used to analyze the expression of WWC3, phosphorylated LATS1, YAP, p-YAP, CTGF and cyclin E in VSMCs, following transfection with WWC3 and treatment with PDGF-BB. (B) VSMCs were transfected with the WWC3 plasmid and subsequently treated with PDGF-BB. A dual-luciferase reporter assay was used to determine the transcriptional activity of TEA domain transcription factors. (C) An MTT assay was used to assess cell proliferation. (D) A scratch wound healing assay was performed and (E) analyzed, and a (F) Transwell chamber assay was performed and (G) analyzed in order to determine cell migration. The results are presented as the mean \pm standard deviation of three independent experiments. * $\mathrm{P}<0.05$. VSMCs, vascular smooth muscle cells; WWC3, WWC family member 3; PDGF-BB, platelet-derived growth factor BB; YAP, YY1-associated protein; p-YAP, phosphorylated YAP; p-LATS1, phosphorylated large tumor suppressor kinase 1; CTGF, connective tissue growth factor; NC, negative control.

treatment with PDGF-BB did not increase transcriptional activity (Fig. 3B). The results demonstrated that enhanced levels of VSMC proliferation and migration were associated with WWC3 activity following treatment with PDGF-BB, and WWC3 inhibited the proliferation and migration of VSMC cells via the Hippo signaling pathway.

WWC3 expression inhibits the translocation of YAP to the nucleus in VSMCs. Numerous studies have demonstrated that the key process associated with the activation of the Hippo signaling pathway is YAP translocation to the nucleus. In the present study, the expression of YAP was revealed to be enhanced following treatment with PDGF-BB. Immunofluorescence staining demonstrated that the location of YAP in the nucleus was markedly increased following treatment with PDGF-BB, whereas the level of nuclear YAP was suppressed following overexpression of WWC3. Following PDGF-BB treatment, YAP expression in VSMC cells that did not overexpress WWC3 was demonstrated to be more predominantly localized in the nucleus, compared with VSMC cells overexpressing WWC3 (Fig. 4A). Western blot analyses of the cytoplasmic and nuclear expression of YAP in VSMCs overexpressing WWC3, following treatment with PDGF-BB, demonstrated the same result (Fig. 4B).
These results suggested that WWC3 regulated the localization of YAP in VSMCs following treatment with PDGF-BB, suppressed YAP translocation into the nucleus and suppressed the expression of downstream genes in the Hippo signaling pathway.

WWC3 regulates the Hippo signaling pathway via interaction with LATS1. Immunoprecipitation analyses were performed, and it was demonstrated via immunofluorescence staining that WWC3 and LATS1 were colocalized in the cytoplasm (Fig. 5A). Additionally, it was revealed that WWC3 may interact with LATS1 (Fig. 5B). In order to further investigate the interaction between WWC3 and LATS1, a WWC3 mutant plasmid with deletion of the WW domain was constructed (Fig. 5C). The results of the immunoprecipitation analyses demonstrated that WWC3 was no longer able to interact with LATS1 in the absence of the WW domain (Fig. 5D). Furthermore, western blot analysis revealed that the expression levels of p-LATS1, p-YAP, YAP, CTGF and cyclin E in cells transfected with WWC3 with a deletion of the WW domain (WWC3- $\triangle \mathrm{WW}$ ) did not markedly alter compared with the control group (Fig. 5E). These results suggested that WWC3 may regulate the Hippo signaling pathway via interaction with LATS1. 


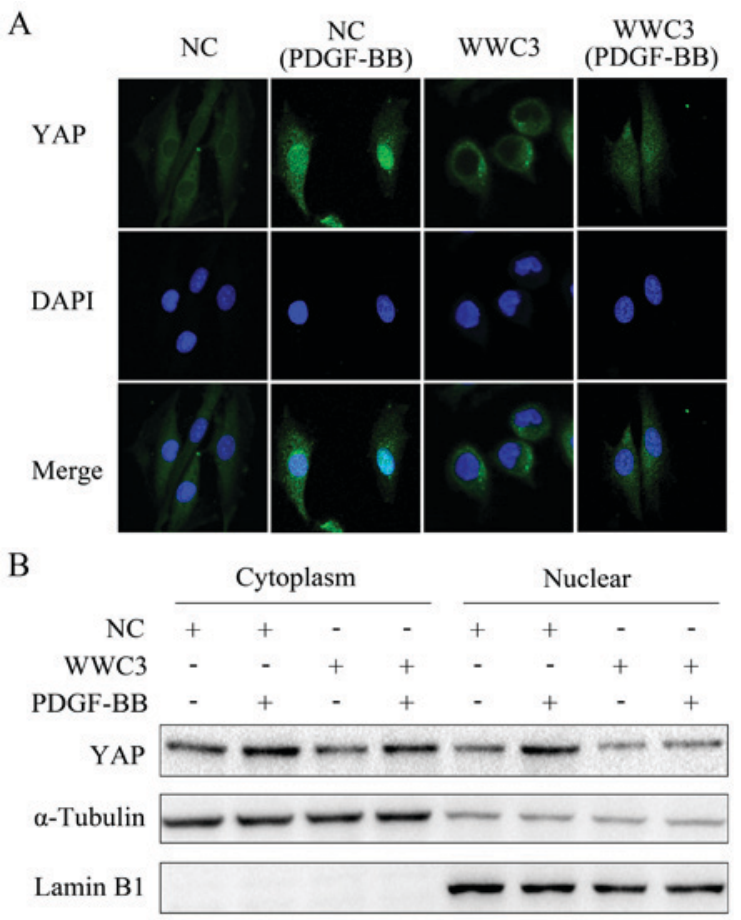

Figure 4. WWC3 suppresses the translocation of YAP to the nucleus in VSMCs treated with PDGF-BB. (A) Immunofluorescent staining of YAP in VSMCs with transfected WWC3 following treatment with PDGF-BB (magnification, x400). (B) Western blot analyses of the cytoplasmic and nuclear expression of YAP in VSMCs with overexpression of WWC3 following treatment with PDGF-BB. $\alpha$-tubulin and lamin B1 were used as cytoplasmic and nuclear markers, respectively. The results are presented as the mean \pm standard deviation of three independent experiments. VSMCs, vascular smooth muscle cells; WWC3, WWC family member 3; PDGF-BB, platelet-derived growth factor BB; YAP, YY1-associated protein; NC, negative control.

\section{Discussion}

The role of the Hippo signaling pathway in cell development and tumorigenesis has been extensively studied; (4-6) however, other studies have suggested that it has an important role in the formation and development of cardiovascular system (11-13). A number of studies have also demonstrated that knockdown of LATS2 and MST1/2 results in heart overgrowth and cardiomyocyte proliferation (11). Further studies have revealed that the Hippo signaling effector YAP is involved in the development of the cardiovascular system and is an important regulator of cardiomyocyte proliferation, cardiac morphogenesis, myocardial trabeculation and vascular development $(19,20)$. Furthermore, numerous animal models of cardiovascular disease have demonstrated that MST, in addition to a number of LATS targets (including microRNAs), are over-activated, and the proliferation and migration of VSMCs is subsequently increased, eventually resulting in vascular remodeling (21-24). However, the study of the Hippo pathway in vascular remodeling remains insufficient; associated factors and mechanisms remain to be determined and thus require further investigation.

The WWCs protein family, which has a highly evolutionarily-conserved molecular structure, may form homodimeric and heterodimeric complexes in order to affect the function and activity of themselves and other molecules (16). A study using 293T cells demonstrated that, with regards to structural similarities, all WWC proteins are able to interact with LATS kinases to negatively regulate the Hippo signaling pathway via activation of LATS kinases (16). At present, the majority of studies have focused on the clinical significance and biological roles of WWC1 in human cancer; thus, whether WWC3 has an association with disease pathology remains largely undetermined. To the best of the authors' knowledge, the present study is the first to reveal that WWC3 expression is downregulated in VSMCs during neointimal hyperplasia following injury (treatment with PDGF-BB or balloon injury), the activity of the Hippo signaling pathway is suppressed post-injury, YAP translocation to the nucleus is increased, and the proliferation and migration of VSMCs is enhanced post-injury. Furthermore, the present study revealed that knockdown of WWC3 may inactivate LATS1, increase the phosphorylation and nuclear translocation of YAP, suppress the activity of the Hippo pathway and enhance the expression of downstream genes, which subsequently enhances the proliferation and migration of VSMCs. These results were consistent with the results obtained following overexpression of WWC3.

YAP, a multifunctional transcriptional coactivator that predominantly functions as an oncoprotein to promote cell proliferation and migration, is the primary effector of the Hippo signaling pathway (25). It was previously demonstrated that cardiac/SMC-specific YAP knockout mice exhibited marked cardiac defects and vascular abnormalities (26). YAP expression is increased during the VSMC phenotypic switch from a contractile to a synthetic state, induced by PDGF-BB stimulation during vessel injury. In addition, YAP overexpression suppresses the expression of VSMC marker genes, and enhances the proliferation and migration of VSMCs $(12,13)$. YAP knockout inhibits VSMC phenotypic modulation otherwise induced by arterial injury, and may attenuate neointimal formation (12). Overexpression of MST1, a negative regulator 


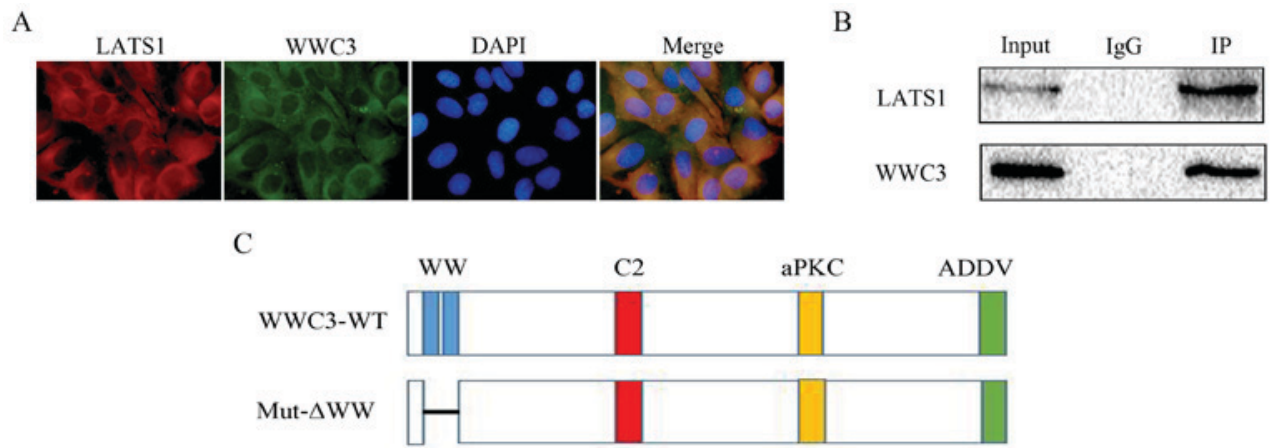

D
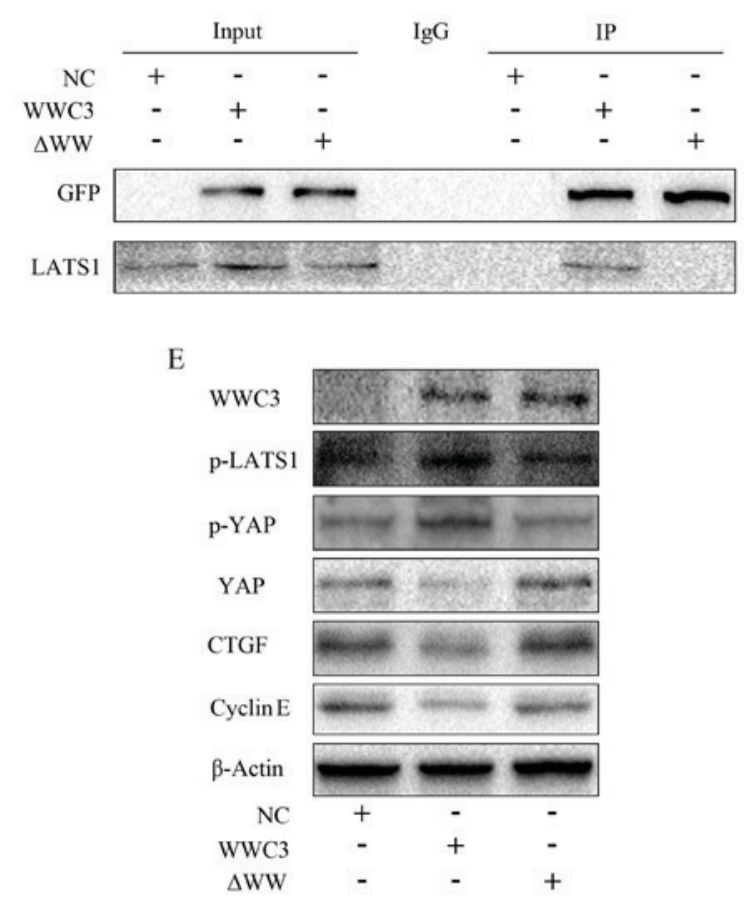

Figure 5. WWC3 regulates the Hippo signaling pathway via interaction with LATS1. (A) Colocalization of WWC3 and LATS1 was observed using immunofluorescence staining (magnification, $\mathrm{x} 400$ ). (B) Immunoprecipitation demonstrated that WWC3 interacted with LATS1 at the endogenous level. WWC 3 is the pulled down protein. (C) Schematic diagram of the structure of pEGFP-C2-WWC3 WT and pEGFP-C2- $\Delta W W$. (D) Immunoprecipitation results demonstrated that WWC3 did not interact with LATS1 following transfection with an empty plasmid and WWC3- $\Delta W W$. (E) The expression of proteins associated with the Hippo signaling pathway following transfection with an empty plasmid, WWC3 or WWC3- $\Delta$ WW. WWC3, WWC family member 3; PDGF-BB, platelet-derived growth factor BB; YAP, YY1-associated protein; p-YAP, phosphorylated YAP; LATS1, large tumor suppressor kinase 1; p-LATS1, phosphorylated LATS1; CTGF, connective tissue growth factor; NC, negative control; IP, immunoprecipitation; IgG, immunoglobulin G; Mut, mutant; $\Delta \mathrm{WW}$, deletion of WW domain; EGFP, enhanced green fluorescent protein; ADDV, C-terminal PDZ-binding motif; aPKC, atypical protein kinase C.

of YAP, may suppress neointimal formation in balloon-injured rat carotid arteries (23). Such studies have revealed various functions of YAP and have demonstrated that the most important regulatory step of the Hippo pathway is the translocation of YAP to the nucleus. The present study demonstrated that injury enhanced the total expression of YAP; however, WWC3 overexpression reduced the expression of YAP and suppressed YAP nuclear import, subsequently suppressing the expression of downstream genes that are associated with cell proliferation and migration. These results suggested that the suppression of YAP nuclear import via WWC may serve as a novel therapeutic target for the treatment of vascular diseases.

LATS1 is considered to be a tumor suppressor gene and may inhibit the proliferation and differentiation of tumor cells, and induce cellular apoptosis via downregulation of the primary effector of the Hippo signaling pathway, YAP (27). LATS1/2 directly interact with YAP and phosphorylate YAP at serine 127 by targeting the HXRXXS motifs in YAP, and subsequently enhancing YAP cytoplasmic sequestration, which suppresses the activity of transcription factors (28). In addition, LATS1/2 also phosphorylate YAP at serine 381, which results in the subsequent phosphorylation of YAP by casein kinase 1, and the recruitment of the E3 ubiquitin ligase, resulting in YAP polyubiquitylation and eventual degradation (29). A recent study demonstrated that LATS1 is inactivated in vascular remodeling, and that LATS1 phosphorylation may markedly suppress vascular remodeling (30). In the present study, it was revealed that WWC3 affects the phosphorylation of LATS1, colocalizes with LATS1, and interacts with LATS1. In the present study, a mutant plasmid of WWC3 with deletion of the WW domain was constructed, and it was demonstrated that WWC3 no longer interacted with LATS1 following WW domain deletion. Furthermore, the levels of p-LATS1, p-YAP, YAP, CTGF and cyclin E did not significantly alter 
following transfection with WWC3- $\Delta \mathrm{WW}$. Therefore, it may be hypothesized that the regulatory effect of WWC3 on the Hippo pathway is dependent on the WWC3-LATS1 interaction via the WW domain.

In conclusion, the present study demonstrated that WWC3 is able to interact with LATS1 to upregulate the Hippo-YAP signaling pathway and suppress the proliferation and migration of VSMCs. This may provide novel therapeutic targets for the prevention and treatment of vascular remodeling diseases, including atherosclerotic diseases, vascular restenosis and hypertension. Further studies are required to investigate the underlying mechanisms regarding the association between injury and the suppression of WWC3 expression.

\section{Acknowledgements}

The authors would like to thank Dr Enhua Wang for assistance in the writing of the present manuscript, and Dr Joachim Kremerskothen for the provision of the WWC3 wild-type and mutant plasmids.

\section{References}

1. Osherov AB, Gotha L, Cheema AN, Qiang BP and Strauss BH: Proteins mediating collagen biosynthesis and accumulation in arterial repair: Novel targets for anti-restenosis therapy. Cardiovasc Res 91: 16-26, 2011.

2. Rodriguez-Menocal L, St-Pierre M, Wei Y, Khan S, Mateu D, Calfa M, Rahnemai-Azar AA, Striker G, Pham SM and Vazquez-Padron RI: The origin of post-injury neointimal cells in the rat balloon injury model. Cardiovasc Res 81: 46-53, 2009.

3. Zhang L, Yang S, Wennmann DO, Chen Y, Kremerskothen J and Dong J: KIBRA: In the brain and beyond. Cell Signal 26: 1392-1399, 2014

4. Buttitta LA and Edgar BA: How size is controlled: From hippos to yorkies. Nat Cell Biol 9: 1225-1227, 2007.

5. Pan D: Hippo signaling in organ size control. Genes Dev 21: 886-897, 2007.

6. Mo JS, Park HW and Guan KL: The Hippo signaling pathway in stem cell biology and cancer. EMBO Rep 15: 642-656, 2014.

7. Dong J, Feldmann G, Huang J, Wu S, Zhang N, Comerford SA, Gayyed MF, Anders RA, Maitra A and Pan D: Elucidation of a universal size control mechanism in Drosophila and mammals. Cell 130: 1120-1133, 2007.

8. Harvey KF, Zhang X and Thomas DM: The Hippo pathway and human cancer. Nat Rev Cancer 13: 246-257, 2013.

9. Han Q, Lin X, Zhang X, Jiang G, Zhang Y, Miao Y, Rong X, Zheng X, Han Y, Han X, et al: WWC3 regulates the Wnt and Hippo pathways via Dishevelled proteins and large tumour suppressor 1, to suppress lung cancer invasion and metastasis. J Pathol 242: 435-447, 2017.

10. Doherty TM, Shah PK and Rajavashisth TB: Cellular origins of atherosclerosis: Towards ontogenetic endgame? FASEB J 17: 592-597, 2003.

11. Heallen T, Zhang M, Wang J, Bonilla-Claudio M, Klysik E, Johnson RL and Martin JF: Hippo pathway inhibits Wnt signaling to restrain cardiomyocyte proliferation and heart size. Science 332: 458-461, 2011.

12. Wang X, Hu G, Gao X, Wang Y, Zhang W, Harmon EY, Zhi X, Xu Z, Lennartz MR, Barroso M, et al: The induction of yes-associated protein expression after arterial injury is crucial for smooth muscle phenotypic modulation and neointima formation. Arterioscler Thromb Vasc Biol 32: 2662-2669, 2012

13. Xie C, Guo Y, Zhu T, Zhang J, Ma PX and Chen YE: Yap1 protein regulates vascular smooth muscle cell phenotypic switch by interaction with myocardin. J Biol Chem 287: 14598-14605, 2012.
14. Genevet A, Wehr MC, Brain R, Thompson BJ and Tapon N: Kibra is a regulator of the Salvador/Warts/Hippo signaling network. Dev Cell 18: 300-308, 2010.

15. Xiao L, Chen Y, Ji M, Volle DJ, Lewis RE, Tsai MY and Dong J: KIBRA protein phosphorylation is regulated by mitotic kinase aurora and protein phosphatase 1. J Biol Chem 286: 36304-36315, 2011.

16. Wennmann DO, Schmitz J, Wehr MC, Krahn MP, Koschmal N, Gromnitza S, Schulze U, Weide T, Chekuri A, Skryabin BV, et al: Evolutionary and molecular facts link the WWC protein family to hippo signaling. Mol Biol Evol 31: 1710-1723, 2014.

17. Wang TR, Yang G and Liu GN: DNA enzyme ED5 depletes egr-1 and inhibits neointimal hyperplasia in rats. Cardiology 125: 192-200, 2013

18. Zhang W, Gao Y, Li P, Shi Z, Guo T, Li F, Han X, Feng Y,Zheng C, Wang Z, et al: VGLL4 functions as a new tumor suppressor in lung cancer by negatively regulating the YAP-TEAD transcriptional complex. Cell Res 24: 331-343, 2014.

19. Morin-Kensicki EM, Boone BN, Howell M, Stonebraker JR, Teed J, Alb JG, Magnuson TR, O'Neal W and Milgram SL: Defects in yolk sac vasculogenesis, chorioallantoic fusion, and embryonic axis elongation in mice with targeted disruption of yap65. Mol Cell Biol 26: 77-87, 2006.

20. von Gise A, Lin Z, Schlegelmilch K, Honor LB, Pan GM, Buck JN, Ma Q, Ishiwata T, Zhou B, Camargo FD and Pu WT: YAP1, the nuclear target of Hippo signaling, stimulates heart growth through cardiomyocyte proliferation but not hypertrophy. Proc Natl Acad Sci USA 109: 2394-2399, 2012.

21. Yamamoto S, Yang G, Zablocki D, Liu J, Hong C, Kim SJ, Soler S, Odashima M, Thaisz J, Yehia G, et al: Activation of mst 1 causes dilated cardiomyopathy by stimulating apoptosis without compensatory ventricular myocyte hypertrophy. J Clin Invest 111: 1463-1474, 2003.

22. Odashima M, Usui S, Takagi H, Hong C, Liu J, Yokota M and Sadoshima J: Inhibition of endogenous mst1 prevents apoptosis and cardiac dysfunction without affecting cardiac hypertrophy after myocardial infarction. Circ Res 100: 1344-1352, 2007.

23. Ono H, Ichiki T, Ohtsubo H, Fukuyama K, Imayama I, Hashiguchi Y, Sadoshima J and Sunagawa K: Critical role of Mst1 in vascular remodeling after injury. Arterioscler Thromb Vasc Biol 25: 1871-1876, 2005.

24. Liu X, Cheng Y, Chen X, Yang J, Xu L and Zhang C: MicroRNA-31 regulated by the extracellular regulated kinase is involved in vascular smooth muscle cell growth via large tumor suppressor homolog 2. J Biol Chem 286: 42371-42380, 2011.

25. Sudol M: Yes-associated protein (YAP65) is a proline-rich phosphoprotein that binds to the $\mathrm{SH} 3$ domain of the Yes proto-oncogene product. Oncogene 9: 2145-2152, 1994.

26. Wang Y, Hu G, Liu F, Wang X, Wu M, Schwarz J and Zhou J: Deletion of yes-associated protein (YAP) specifically in cardiac and vascular smooth muscle cells reveals a crucial role for YAP in mouse cardiovascular development. Circ Res 114: 957-965, 2014.

27. Zhao B, Lei QY and Guan KL: The Hippo-YAP pathway: New connections between regulation of organ size and cancer. Curr Opin Cell Biol 20: 638-646, 2008.

28. Zhao B, Wei X, Li W, Udan RS, Yang Q, Kim J, Xie J, Ikenoue T, $\mathrm{Yu} \mathrm{J}, \mathrm{Li} \mathrm{L}$, et al: Inactivation of YAP oncoprotein by the Hippo pathway is involved in cell contact inhibition and tissue growth control. Genes Dev 21: 2747-2761, 2007.

29. Zhao B, Tumaneng K and Guan KL: The Hippo pathway in organ size control, tissue regeneration and stem cell self-renewal. Nat Cell Biol 13: 877-883, 2011.

30. Kudryashova TV, Goncharov DA, Pena A, Kelly N, Vanderpool R, Baust J, Kobir A, Shufesky W, Mora AL, Morelli AE, et al: HIPPO-integrin linked kinase cross-talk controls self-sustaining proliferation and survival in pulmonary hypertension. Am J Respir Crit Care Med 194: 866-877, 2016.

This work is licensed under a Creative Commons Attribution-NonCommercial-NoDerivatives 4.0 International (CC BY-NC-ND 4.0) License. 